\title{
Contrasting Two Transformation-Based Methods for Obtaining Absolute Extrema
}

\author{
D. F. M. Torres ${ }^{1}$ And G. LeItmann ${ }^{2}$
}

\begin{abstract}
In this note we contrast two transformation-based methods to deduce absolute extrema and the corresponding extremizers. Unlike variation-based methods, the transformation-based ones of Carlson and Leitmann and the recent one of Silva and Torres are direct in that they permit obtaining solutions by inspection.
\end{abstract}

Mathematics Subject Classification 2000: 49J15, 49M30.

Key Words. Optimal Control, direct methods, Calculus of Variations, absolute extrema, invariance, symmetry.

\section{Introduction}

In the mid 1960's a direct method for the problems of the calculus of variations, which permits one to obtain absolute extremizers directly, without using variational methods, was introduced by Leitmann (Ref. 9). Since then, this direct method has been extended and applied to a variety of problems (see e.g. Refs. 1, 3, 4, 10). A different but related direct approach to problems of optimal control, based on the variational symmetries of the problem (cf. Refs. 7, 8), was recently introduced by Silva and Torres (Ref. 11). The emphasis in Ref. 11 has been on showing the differences and similarities between the proposed method and that suggested by Leitmann. In order to illustrate the relation between these two methods, only examples capable of

\footnotetext{
${ }^{1}$ Associate Professor in the Department of Mathematics, University of Aveiro, Aveiro, Portugal.

${ }^{2}$ Professor in the Graduate School, College of Engineering, University of California, Berkeley, California.
} 
treatment by both methods were presented in Ref. 11. In this note, we discuss some differences between the method of Carlson and Leitmann (C/L) and Silva and Torres $(\mathrm{S} / \mathrm{T})$. In particular, we show how one succeeds when the other does not.

\section{The Invariant Transformation Method of $\mathrm{S} / \mathrm{T}^{3}$}

Let us consider the problem of optimal control in Lagrange form: minimize an integral

$$
I[x(\cdot), u(\cdot)]=\int_{a}^{b} L(t, x(t), u(t)) d t
$$

subject to a control system

$$
\dot{x}(t)=\varphi(t, x(t), u(t)) \quad \text { a.e. on }[a, b],
$$

together with appropriate boundary conditions and constraint on the values of the control variables:

$$
x(a)=x_{a}, \quad x(b)=x_{b}, \quad u(t) \in \Omega .
$$

The Lagrangian $L(\cdot, \cdot, \cdot)$ is a real function assumed to be continuously differentiable in $[a, b] \times \mathbb{R}^{n} \times \mathbb{R}^{m} ; t \in \mathbb{R}$ is the independent variable; $x(\cdot)$ : $[a, b] \rightarrow \mathbb{R}^{n}$ the vector of state variables; $u(\cdot):[a, b] \rightarrow \Omega \subseteq \mathbb{R}^{m}$ the vector of controls, assumed to be a piecewise continuous function; and $\varphi(\cdot, \cdot, \cdot):[a, b] \times \mathbb{R}^{n} \times \mathbb{R}^{m} \rightarrow \mathbb{R}^{n}$ the derivative function, assumed to be a continuously differentiable vector function. When $\Omega$ is an open set (it may be all Euclidean space $\Omega=\mathbb{R}^{m}$ ), problem (11)-(3) can be studied using the classical techniques of the Calculus of Variations. Optimal Control Theory includes the classical Calculus of Variations and generalizes the theory by dealing with the cases where $\Omega$ is not an open set.

The application of the invariant transformation method (Ref. 11) depends on the existence of a sufficiently rich family of invariance transformations (variational symmetries). The reader interested on the study of variational symmetries is referred to Refs. 8, 12, 13 and references therein.

Definition 2.1 Let $h^{s}$ be a s-parameter family of $C^{1}$ mappings satisfying:

$$
\begin{aligned}
& h^{s}(\cdot, \cdot, \cdot):[a, b] \times \mathbb{R}^{n} \times \Omega \longrightarrow \mathbb{R} \times \mathbb{R}^{n} \times \mathbb{R}^{m}, \\
& h^{s}(t, x, u)=\left(t^{s}(t, x, u), x^{s}(t, x, u), u^{s}(t, x, u)\right), \\
& h^{0}(t, x, u)=(t, x, u), \quad \forall(t, x, u) \in[a, b] \times \mathbb{R}^{n} \times \Omega .
\end{aligned}
$$

\footnotetext{
${ }^{3}$ Throughout this Note, the notation conforms to that used in the references.
} 
If there exists a function $\Phi^{s}(t, x, u) \in C^{1}\left([a, b], \mathbb{R}^{n}, \Omega ; \mathbb{R}\right)$ such that

$$
L \circ h^{s}(t, x(t), u(t)) \frac{d}{d t} t^{s}(t, x(t), u(t))=L(t, x(t), u(t))+\frac{d}{d t} \Phi^{s}(t, x(t), u(t))
$$

and

$$
\frac{d}{d t} x^{s}(t, x(t), u(t))=\varphi \circ h^{s}(t, x(t), u(t)) \frac{d}{d t} t^{s}(t, x(t), u(t))
$$

for all admissible pairs $(x(\cdot), u(\cdot))$, then (11) -(2) is said to be invariant under the transformations $h^{s}(t, x, u)$ up to $\Phi^{s}(t, x, u)$; and the transformations $h^{s}(t, x, u)$ are said to be a variational symmetry of (11) -(2).

The method proposed in Ref. 11 is based on a very simple idea. Given an optimal control problem, one begins by determining its invariance transformations according to Definition 2.1. With respect to this, the tools developed in Refs. 7, 8 are useful. Applying the parameter-invariance transformations, we embed our problem into a parameter-family of optimal control problems. Given the invariance properties, if we are able to solve one of the problems of this family, we also get the solution to our original problem (or to any other problem of the same family) from the invariant transformations. In section 4 we give an example which shows that the Invariant Transformation Method (Ref. 11) is more general than the earlier C/L transformation method in the case of optimal control problems.

\section{The Direct Solution Method of C/L}

Since this method is fully discussed in readily available references, e.g. Refs. 1, 3, 4, 9, 10, many in this journal, we shall only recall that the $\mathrm{C} / \mathrm{L}$ transformation based method is applicable to problems in the Calculus of Variations format: minimize an integral

$$
I[x(\cdot)]=\int_{a}^{b} F(t, x(t), \dot{x}(t)) d t
$$

with given end conditions

$$
x(a)=x_{a}, \quad x(b)=x_{b} .
$$

If one wishes to solve an optimal control problem (1)-(3), the "elimination" of $u(t)$ in favor of a function of $t, x(t), \dot{x}(t)$ must be possible. As illustrated in section 4, this may fail even if the Implicit Function Theorem is satisfied. 
Both the $\mathrm{S} / \mathrm{T}$ and the $\mathrm{C} / \mathrm{L}$ methods are predicated on posing a problem "equivalent" to the original problems (11)-(3) and (6)-(17), respectively. Thus, these methods are useful only if the solution of the "equivalent" problem is directly obtainable, i.e., by inspection. There is, at present, no result assuring that this can be done in general for the $\mathrm{S} / \mathrm{T}$ method. However, for the $\mathrm{C} / \mathrm{L}$ method, at least in the scalar $x$ case, it has been shown in Ref. 5 and generalized to open-loop differential games in Ref. 2, that the "equivalent" problem always has a minimizing solution obtained by inspection. The conditions sufficient for this result are convexity of integrand $F(t, x(t), \dot{x}(t))$ with respect to $\dot{x}(t)$, and existence of a so-called "field of extremals". Indeed, no matter what the integrand of the original problem is, provided the conditions above are met, the absolute minimizer of the equivalent problem is always a constant.

\section{Example 1}

The advantage of the invariant transformation method when compared with the earlier transformation method is that one can apply it directly to control systems whereas the method of $\mathrm{C} / \mathrm{L}$ requires that the control $u(t)$ can be expressed as a sufficiently smooth function of $t, x(t), \dot{x}(t)$, e.g. such that the integrand be continuous in $x(t)$ and $\dot{x}(t)$. Here we use the invariant transformation method of $\mathrm{S} / \mathrm{T}$ to solve a simple optimal control problem that is not covered by the classical theory of the Calculus of Variations and which can not be solved by the previous transformation method.

Consider the global minimum problem

$$
\begin{gathered}
I\left[u_{1}(\cdot), u_{2}(\cdot)\right]=\int_{0}^{1}\left(u_{1}(t)^{2}+u_{2}(t)^{2}\right) d t \longrightarrow \min \\
\dot{x}_{1}(t)=\exp \left(u_{1}(t)\right)+u_{1}(t)+u_{2}(t), \\
\dot{x}_{2}(t)=u_{2}(t), \\
x_{1}(0)=0, \quad x_{1}(1)=2, \quad x_{2}(0)=0, \quad x_{2}(1)=1, \\
u_{1}(t), u_{2}(t) \in \Omega=[-1,1] .
\end{gathered}
$$

We apply the procedure introduced in Ref. 11 and briefly described in section 2. First we notice that problem (8) is variationally invariant according 
to Definition 2.1 under the one-parameter transformations ${ }^{4}$

$$
x_{1}^{s}=x_{1}+s t, \quad x_{2}^{s}=x_{2}+s t, \quad u_{2}^{s}=u_{2}+s \quad\left(t^{s}=t \text { and } u_{1}^{s}=u_{1}\right) .
$$

To prove this, we need to show that both the functional integral $I[\cdot]$ and the control system stay invariant under the $s$-parameter transformations (9). This is easily seen by direct calculations. We begin by showing (4):

$$
\begin{aligned}
I^{s}\left[u_{1}^{s}(\cdot), u_{2}^{s}(\cdot)\right] & =\int_{0}^{1}\left(u_{1}^{s}(t)\right)^{2}+\left(u_{2}^{s}(t)\right)^{2} d t \\
& =\int_{0}^{1} u_{1}(t)^{2}+\left(u_{2}(t)+s\right)^{2} d t \\
& =\int_{0}^{1} u_{1}(t)^{2}+u_{2}(t)^{2}+\frac{d}{d t}\left(s^{2} t+2 s x_{2}(t)\right) d t \\
& =I\left[u_{1}(\cdot), u_{2}(\cdot)\right]+s^{2}+2 s .
\end{aligned}
$$

We remark that $\Phi^{s}\left(t, x_{2}\right)=s^{2} t+2 s x_{2}$ and that $I^{s}[\cdot]$ and $I[\cdot]$ have the same minimizers: adding a constant $s^{2}+2 s$ to the functional $I[\cdot]$ does not change the minimizer of $I[\cdot]$. It remains to prove $(\underline{5})$ :

$$
\begin{aligned}
\frac{d}{d t}\left(x_{1}^{s}(t)\right) & =\frac{d}{d t}\left(x_{1}(t)+s t\right)=\dot{x}_{1}(t)+s=\exp \left(u_{1}(t)\right)+u_{1}(t)+u_{2}(t)+s \\
& =\exp \left(u_{1}^{s}(t)\right)+u_{1}^{s}(t)+u_{2}^{s}(t) \\
\frac{d}{d t}\left(x_{2}^{s}(t)\right) & =\frac{d}{d t}\left(x_{2}(t)+s t\right)=\dot{x}_{2}(t)+s=u_{2}(t)+s \\
& =u_{2}^{s}(t) .
\end{aligned}
$$

Conditions (10) and (11) prove that problem (8) is invariant under the $s$-parameter transformations (9) up to the gauge term $\Phi^{s}=s^{2} t+2 s x_{2}$. Using the invariance transformations (9), we generalize problem (8) to a $s$-parameter family of problems, $s \in \mathbb{R}$, which include the original problem

\footnotetext{
${ }^{4} \mathrm{~A}$ computer algebra package that can be used to find the invariance transformations (see Refs. 7, 8) is available from the Maple Application Center at http://www.maplesoft. com/applications/app_center_view . aspx?AID=1983
} 
for $s=0$ :

$$
\begin{gathered}
I^{s}\left[u_{1}^{s}(\cdot), u_{2}^{s}(\cdot)\right]=\int_{0}^{1}\left(u_{1}^{s}(t)\right)^{2}+\left(u_{2}^{s}(t)\right)^{2} d t \rightarrow \min \\
\dot{x}_{1}^{s}(t)=\exp \left(u_{1}^{s}(t)\right)+u_{1}^{s}(t)+u_{2}^{s}(t), \\
\dot{x}_{2}(t)=u_{2}^{s}(t), \\
x_{1}^{s}(0)=0, \quad x_{1}^{s}(1)=2+s, \quad x_{2}^{s}(0)=0, \quad x_{2}^{s}(1)=1+s, \\
u_{1}^{s}(t) \in[-1,1], \quad u_{2}^{s}(t) \in[-1+s, 1+s] .
\end{gathered}
$$

It is clear that $I^{s} \geq 0$ and that $I^{s}=0$ if $u_{1}^{s}(t)=u_{2}^{s}(t) \equiv 0$. The control equation, the boundary conditions and the constraints on the values of the controls, imply that $u_{1}^{s}(t)=u_{2}^{s}(t) \equiv 0$ is admissible only if $s=-1$ : $x_{1}^{s=-1}(t)=t, x_{2}^{s=-1}(t) \equiv 0$. Hence, for $s=-1$ the global minimum to $I^{s}[\cdot]$ is 0 and the minimizing trajectory is given by

$$
\tilde{u}_{1}^{s}(t) \equiv 0, \quad \tilde{u}_{2}^{s}(t) \equiv 0, \quad \tilde{x}_{1}^{s}(t)=t, \quad \tilde{x}_{2}^{s}(t) \equiv 0 .
$$

Since for any $s$ one has by (10) that $I\left[u_{1}(\cdot), u_{2}(\cdot)\right]=I^{s}\left[u_{1}^{s}(\cdot), u_{2}^{s}(\cdot)\right]-s^{2}-2 s$, we conclude that the global minimum for problem $I\left[u_{1}(\cdot), u_{2}(\cdot)\right]$ is 1 . Thus, using the inverse functions of the variational symmetries (9),

$u_{1}(t)=u_{1}^{s}(t), \quad u_{2}(t)=u_{2}^{s}(t)-s, \quad x_{1}(t)=x_{1}^{s}(t)-s t, \quad x_{2}(t)=x_{2}^{s}(t)-s t$,

the absolute minimizer is

$$
\tilde{u}_{1}(t)=0, \quad \tilde{u}_{2}(t)=1, \quad \tilde{x}_{1}(t)=2 t, \quad \tilde{x}_{2}(t)=t .
$$

This problem cannot be solved by the $\mathrm{C} / \mathrm{L}$ method.

While the existence of a function $h(\cdot)$ such that $u_{1}(t)=h\left(\dot{x}_{1}(t)-\dot{x}_{2}(t)\right)$ is assured by the satisfaction of the Implicit Function Theorem, this is not useful in "eliminating" the control in favor of $t, x(t), \dot{x}(t)$ which is a requirement of the $\mathrm{C} / \mathrm{L}$ method. This is so because $h(\cdot)$ is a solution of a transcendental equation. In addition, since the controls are bounded, even if "elimination" of the control were possible, this would lead to differential constraints of the form briefly discussed in Ref. 6 .

\section{$5 \quad$ Example 2}

Consider the problem of attaining the absolute minimum of integral

$$
I[x(\cdot)]=\int_{a}^{b}\left[\dot{x}^{2}(t)+t \dot{x}(t)\right] d t
$$


with prescribed end conditions

$$
x(a)=x_{a}, \quad x(b)=x_{b} .
$$

This is a problem in the Calculus of Variations format for which the C/L or $\mathrm{S} / \mathrm{T}$ transformation-based method applies. These methods can be applied $a b$ initio to obtain the solution. However, here we shall employ the constructive sufficiency condition embodied in Theorem 7 of Ref. 5 towards that end.

The Euler-Lagrange equation is

$$
\ddot{x}(t)=-\frac{1}{2}
$$

so that

$$
x(t)=-\frac{1}{4} t^{2}+c_{1} t+c_{2}
$$

is the extremal with the constants of integration given by end conditions (13), say $c_{i}=c_{i}^{*}, i=1,2$, and the extremal satisfying (15) is

$$
x^{*}(t)=-\frac{1}{4} t^{2}+c_{1}^{*} t+c_{2}^{*}
$$

which, being the solution of necessary condition (14), is a candidate for the absolute minimizer of (12)-(13).

Now we can readily show that the conditions of Theorem 7 of Ref. 5$]$ are met. Consider the one-parameter family of extremals

$$
\xi(t, \beta)=-\frac{1}{4} t^{2}+c_{1}^{*} t+c_{2}^{*}+\beta .
$$

First of all, there exist a $\beta^{*}$, namely $\beta^{*}=0$, such that

$$
\xi\left(t, \beta^{*}\right)=x^{*}(t)
$$

Secondly,

$$
\frac{\partial \xi(t, \beta)}{\partial \beta} \neq 0,
$$

and finally, the integrand of (12) is convex in $\dot{x}(t)$.

Thus, the extremal (16) is indeed the absolute minimizer of (12)-(13).

Of course, this is precisely the solution obtained by employing the $\mathrm{C} / \mathrm{L}$ method. Indeed, the more general method inherent in Theorem 7 of Ref. 5 was derived using the $\mathrm{C} / \mathrm{L}$ transformation-based method. 


\section{Conclusion}

We have contrasted two transformation-based methods for obtaining absolutely extremizing solutions for two classes of problems. One, dubbed the Carlson/Leitmann method, is applicable to problems in the format of the Calculus of Variations. The other, due to Silva and Torres, is applicable to problems of Optimal Control.

We have shown that it is not always possible to convert an Optimal Control problem into a Calculus of Variations one. Hence, the $\mathrm{C} / \mathrm{L}$ method may fail to apply when the $\mathrm{S} / \mathrm{T}$ succeeds. On the other hand, a classical constructive sufficiency condition, readily derived by the $\mathrm{C} / \mathrm{L}$ method, renders absolute extremizers for specific problems of the Calculus of Variations more directly and succinctly than the $\mathrm{C} / \mathrm{L}$ and $\mathrm{S} / \mathrm{T}$ methods.

\section{References}

1. CARLSON, D. A., An Observation on Two Methods of Obtaining Solutions to Variational Problems, J. Optim. Theory Appl., Vol. 114, pp. 345-361, 2002.

2. CARLSON, D. A., Fields of Extremals and Sufficient Conditions for a Class of Variational Games, Proceed. of 12th International Symposium on Dynamic Games and Applications, Sophia Antipolis, France, 2006, to appear.

3. CARlSOn, D. A., and LEITMANN, G., Coordinate Transformation Method for the Extremization of Multiple Integrals, J. Optim. Theory Appl., Vol. 127, pp. 523-533, 2005.

4. CARlSOn, D. A., and LEITMANN, G., A Direct Method for OpenLoop Dynamic Games for Affine Control Systems. Dynamic games: theory and applications, 37-55, GERAD 25th Anniv. Ser., 10, Springer, New York, 2005.

5. CARLSON, D. A., and LEITMANN, G., Fields of Extremals and Sufficient Conditions for the Simplest Problem of the Calculus of Variations, J. Global Optim., 2007, to appear.

6. CARTIGNY, P., and DEISSENBERG, C., An Extension of Leitmann's Direct Method to Inequality Constraints, Int. Game Theory Rev., Vol. 6, pp. 15-20, 2004. 
7. GOUVEIA, P. D. F., and TORRES, D. F. M., Automatic Computation of Conservation Laws in the Calculus of Variations and Optimal Control, Computational Methods in Applied Mathematics, Vol. 5, pp. 387409, 2005.

8. GOUVEIA, P. D. F., TORRES, D. F. M., and ROCHA, E. A. M., Symbolic Computation of Variational Symmetries in Optimal Control, Control and Cybernetics, Vol. 35, pp. 831-849, 2006.

9. LEITMAnN, G., A Note on Absolute Extrema of Certain Integrals, International Journal of Nonlinear Mechanics, Vol. 2, pp. 55-59, 1967.

10. LeITMAnN, G., Some Extensions to a Direct Optimization Method, J. Optim. Theory Appl., Vol. 111, pp. 1-6, 2001.

11. SILVA, C. J., and TORRES, D. F. M., Absolute Extrema of Invariant Optimal Control Problems, Commun. Appl. Anal., Vol. 10, pp. 503-516, 2006.

12. TORRES, D. F. M., Quasi-Invariant Optimal Control Problems, Port. Math. (N.S.), Vol. 61, pp. 97-114, 2004.

13. TORRES, D. F. M., A Noether Theorem on Unimprovable Conservation Laws for Vector-Valued Optimization Problems in Control Theory, Georgian Mathematical Journal, Vol. 13, pp. 173-182, 2006. 